\title{
Avaliação da qualidade de vida em diferentes setores de uma IES privada
}

\section{Evaluation of quality of life in different sectors in private university}

\author{
Indira Léa Melo Ravagnani \\ Universidade de Franca - UNIFRAN, Franca, Brasil \\ indira87@globo.com \\ Carolina de Freitas Fontes \\ Universidade de Franca - UNIFRAN, Franca, Brasil \\ carolffontes@yahoo.com.br \\ José Eduardo Zaia \\ Universidade de Franca - UNIFRAN, Franca, Brasil \\ zaia@unifran.br \\ Cassiano Merussi Neiva \\ Universidade de Franca - UNIFRAN, Franca, Brasil \\ cmn@fc.unesp.br \\ Cléria Maria Lobo Bittar \\ Universidade de Franca - UNIFRAN, Franca, Brasil \\ cml.bittar@gmail.com \\ Paulo Roberto Veiga Quemelo \\ Universidade de Franca - UNIFRAN, Franca, Brasil \\ pquemelo@unifran.br
}

\section{RESUMO}

OBJETIVO: Avaliar a qualidade de vida dos funcionários de três diferentes setores de uma Instituição de Ensino Superior (IES).

MÉTODOS: Foram avaliados 146 funcionários utilizando o questionário de qualidade de vida SF36. Os funcionários foram divididos em três diferentes grupos de acordo com a função exercida: setor I = limpeza/manutenção; setor II = secretaria; setor III = clínicas/laboratórios.

RESULTADOS: Os resultados encontrados no questionário SF36 apresentaram uma média de 73,49 $\pm 8,75$ pontos, com maior pontuação para os aspectos emocionais $(83,81 \pm 3,1$ pontos), seguido das limitações por aspectos físicos (81,98 $\pm 4,6$ pontos) e capacidade funcional ( $80,83 \pm 2,7$ pontos). Já os itens dor (66,84 $\pm 6,9$ pontos), saúde mental $(66,78 \pm 7,6$ pontos) e vitalidade (59,01 $\pm 1,7$ pontos) apresentam as notas mais baixas. Quando analisado separadamente por setores, o setor I apresentou as notas mais baixas (69,03 \pm 9,9 pontos), principalmente para os quesitos dor $(59,31 \pm 25,7)$, saúde mental $(58,56 \pm 20,6$ pontos $)$ e vitalidade (57,56 $\pm 11,9$ pontos), enquanto que os setores II e III apresentaram em média maior nota 76,61 $\pm 9,1$ e 74,83 $\pm 8,3$, respectivamente. 
CONCLUSÕES: Embora os escores encontrados no SF36 foram razoáveis e não apresentou diferença estatística, o estudo aponta diferentes pontuações obtidas em relação aos setores de trabalho. Talvez a diferença de remuneração, educação e exposição biomecânica, entre os setores, possam explicar as menores notas apresentadas pelo setor I.

PALAVRAS-CHAVE: Qualidade de vida. Percepção. Ergonomia. Trabalho.

\section{ABSTRACT}

OBJECTIVE: To evaluate the quality of life of employees from three different sectors in a university.

METHODS: The 146 employees were evaluated using the quality of life questionnaire SF36. The employees were separated in accordance to activity performed: sector $\mathrm{I}=$ cleaning / maintenance; sector II = secretary, sector III = clinical / lab.

RESULTS: The results found in SF36 showed an average of $73.49 \pm 8.75$ points, with higher scores for the emotional aspects $(83.81 \pm 3.1$ points $)$, followed by physical limitations $(81.98$ \pm 4.6 points $)$ and physical limitations $(80.83 \pm 2.7$ points $)$. The items pain $(66.84 \pm 6.9$ points $)$, mental health $(66.78 \pm 7.6$ points $)$ and vitality $(59.01 \pm 1.7$ points $)$ have the lowest scores. When analyzed separately, the sector I had the lowest scores $(69.03 \pm 9.9$ points $)$, mainly for pain questions $(59.31 \pm 25.7)$, mental health $(58.56 \pm 20.6$ points $)$ and vitality (11.9 \pm 57.56 points), while the sectors II and III showed a higher average score $76.61 \pm 9.1$ and $74.83 \pm 8.3$, respectively.

CONCLUSIONS: Although the scores found in SF36 were reasonable and did not show statistical difference, the study suggests different scores in relation to work sectors. Perhaps the difference in salary, education and biomechanics exposure, between sectors, may explain the lower scores presented by sector I.

KEYWORDS: Quality of life. Perception. Ergonomics. Work.

\section{Introdução}

O termo qualidade de vida oferece um amplo leque de definições, composto de ramificações que envolvem fatores físicos, funcionais, emocionais e até mesmo fatores interpessoais como lazer, família e condições de trabalho (JARDIM; BARRETO; ASSUNÇÃO, 2007).

Devido às inovações tecnológicas e organizacionais o ambiente de trabalho vem passando por constantes mudanças. A relação homem-máquina se transforma pela suposta diminuição das tarefas árduas e penosas, as quais geravam muitos acidentes e doenças profissionais, porém, ainda existem riscos mais sutis que envolvem os aspectos físicos, sociais e mentais que geram diminuição da qualidade de vida dos trabalhadores (OLIVEIRA, 1997).

Desta maneira, a atividade laboral não pode ser vista apenas como um meio de sobrevivência por resultar em remuneração, mas sim como uma maneira de participação efetiva na comunidade, um meio de relações interpessoais e de possibilidades, atuando também como importante fonte de autorrealização e de satisfação para com a vida (RIBEIRO; LEDA, 2004).

Apesar das doenças físicas, como os distúrbios osteomusculares relacionados ao trabalho (DORTs), terem se tornado praticamente uma epidemia, é importante enfatizar que não apenas as 'doenças físicas' estão afetando os trabalhadores. Na União Européia, no ano 
2000, o estresse foi o segundo problema mais comum e frequente encontrado no âmbito laboral. Vale ressaltar que as alterações feitas pelo mundo moderno e tecnológico intensificaram as horas trabalhadas, incluindo mais horas extras e finais de semana podendo atingir altos níveis de fadiga mental (ARTAZCOZ, 2002). Neste contexto, a preocupação com a qualidade de vida, a construção de ambientes de trabalho saudáveis e a adequação das condições de salubridade e convivência são estratégias fundamentais da Promoção de Saúde. Com isso as estratégias de prevenção de doenças atuando transversalmente na busca pela integralidade e visão holística do ser humano vêm crescendo nos últimos anos (BRASIL, 2002).

Em recente estudo publicado com trabalhadores de uma instituição de ensino superior pública foi observado que a qualidade de vida esta relacionada com o ambiente de trabalho, remuneração e plano de carreira (FREITAS; SOUZA; QINTELLA, 2013). Portanto, a qualidade de vida tem relação direta com a sensação de bem-estar mental e espiritual, bem como nas relações interpessoais, representando um conceito amplo que envolve os aspectos físicos, sociais e emocionais (LUGO; GARCIA; GÓMEZ, 2006; FREITAS; SOUZA; QINTELLA, 2013; MACIEL et al., 2013).

Diante do exposto, a avaliação da qualidade de vida dos trabalhadores em diferentes setores mostra-se como uma importante ferramenta para traçar estratégias de promoção de saúde, direcionadas conforme a necessidade de cada local de trabalho. Assim, o objetivo do presente estudo foi avaliar a qualidade de vida dos funcionários de três diferentes setores de uma Instituição de Ensino Superior (IES).

\section{Material e Métodos}

Participaram deste estudo funcionários de três setores de uma instituição privada de ensino superior, cujas funções admitem posturas físicas e remunerações salariais diferentes em função do tipo de trabalho realizado. No setor I $(n=39)$, responsável pela limpeza e manutenção predial, as funções são basicamente realizadas na posição em pé ou agachada. $\mathrm{O}$ setor II $(n=85)$, que representa a parte administrativa, os funcionários exercem suas atividades laborais principalmente sentados, com o uso de computadores. O setor III $(n=22)$, que representa os laboratórios e clínicas, apresenta funções variadas, sendo as mesmas, muitas vezes, realizadas nas posições sentada e em pé, devido às distintas tarefas, como a análise em microscópios, a esterilização de equipamentos, uso de computadores, entre outros.

O quadro total de funcionários desses setores na Instituição era de 453 no período que foi realizado a pesquisa. Foram convidados a participar da pesquisa todos os funcionários, sendo que 152 questionários foram respondidos. Dentre eles, seis estavam preenchidos inadequadamente, sendo excluídos da pesquisa. Desta forma, a amostra foi constituída por 146 funcionários, equivalente a $32,2 \%$ da população, e resultando em uma margem de erro de $6,7 \%$.

A coleta de dados foi realizada nos respectivos postos de trabalho de cada funcionário. Este procedimento ocorreu por meio de questionário multidimensional formado por 36 itens, distribuídos em oito domínios: capacidade funcional (dez itens), aspectos físicos (quatro itens), dor (dois itens), estado geral da saúde (cinco itens), vitalidade (quatro itens), aspectos sociais (dois itens), aspectos emocionais (três itens), saúde mental (cinco itens) e mais uma questão de avaliação comparativa entre as condições de saúde atual e a de um ano atrás. $\mathrm{O}$ SF36 avalia tanto os aspectos negativos da saúde (doença ou enfermidade), como os aspectos positivos (bem-estar) (MARTINEZ, 2002). O SF36 representa definições múltiplas da saúde, inclusive função e deficiência orgânica, desconforto e bem-estar, relatórios objetivos e reclamações subjetivas, de solenidade-avaliação favorável e desfavorável da condição de saúde (CICONELLI et al., 1999). O questionário SF36 foi desenvolvido nos Estados Unidos e 
mostrou confiabilidade e validade, e já foi utilizado em mais de 40 países (LUGO, GARCIA; GÓMEZ, 2006).

As coletas foram realizadas no período da manhã e noite, durante cinco semanas, entre os meses de maio e junho de 2011. Os questionários foram entregues aos funcionários que foram esclarecidos sobre seu preenchimento e auxiliados quanto às dúvidas que por ventura surgiram. A coleta foi realizada em períodos diferentes devido à troca de turnos dos funcionários.

Os dados foram tabulados em planilha do Microsoft Office Excel para verificar a média, desvio padrão e porcentagem. Para comparar a qualidade de vida entre os setores analisados foi utilizado o programa GraphPad Prism, utilizando o teste de Kolmogorov Smirnov, para verificação de distribuição normal entre os domínios. Para a comparação dos resultados que apresentaram distribuição normal foi utilizado o teste análise de variância (ANOVA). Para os dados que não apresentaram distribuição normal foi utilizado o teste de Kruskal-Wallis, com pós-teste de Tukey, considerando significativas quando o $p<0,05$.

O estudo foi aprovado pelo Comitê de Ética em Pesquisa da Universidade de Franca, de acordo com a Resolução 196/96, do Conselho Nacional de Saúde (CNS), sob protocolo de número 0069/10.

\section{Resultados}

Dos 146 participantes, 113 eram mulheres e 33 homens, correspondendo a 77,39\% do sexo feminino e $22,61 \%$ do sexo masculino. A idade dos participantes variou de 18 a 59 anos de idade, sendo a média de 36,48 $\pm 10,63$ anos.

Os resultados encontrados no questionário SF36 apresentaram uma média geral de $73,49 \pm 8,75$ pontos. Quando analisado separadamente por setores, o setor I apresentou as notas mais baixas $(69,03 \pm 9,9$ pontos), enquanto que os setores II e III apresentaram em média maior nota $76,61 \pm 9,1$ e 74,83 $\pm 8,3$, respectivamente, porém, não apresentaram diferença estatística, como mostra a tabela 1.

Tabela 1 - Pontuação para os diferentes aspectos do questionário SF36 nos setores analisados

\begin{tabular}{l|c|c|c|c}
\hline \multicolumn{1}{c|}{ SF36 } & Média geral & Setor I & Setor II & Setor III \\
\hline Capacidade Funcional & $80,83 \pm 2,7$ & $78,21 \pm 18,3$ & $83,59 \pm 17,0$ & $80,68 \pm 14,3$ \\
\hline $\begin{array}{l}\text { Limitação por aspectos } \\
\text { físicos }\end{array}$ & $81,98 \pm 4,6$ & $78,21 \pm 31,0$ & $87,06 \pm 21,3$ & $80,68 \pm 30,8$ \\
\hline Dor & $66,84 \pm 6,9$ & $59,31 \pm 25,7$ & $68,36 \pm 23,2$ & $72,86 \pm 24,8$ \\
\hline Estado geral de saúde & $73,10 \pm 7,3$ & $64,74 \pm 20,6$ & $78,15 \pm 19,9$ & $76,41 \pm 17,5$ \\
\hline Vitalidade & $59,01 \pm 1,7$ & $57,56 \pm 11,9$ & $60,84 \pm 12,3$ & $58,64 \pm 11,6$ \\
\hline Aspectos Sociais & $75,55 \pm 0,5$ & $75,32 \pm 23,0$ & $75,20 \pm 25,4$ & $76,14 \pm 26,4$ \\
\hline Aspectos Emocionais & $83,81 \pm 3,1$ & $80,32 \pm 29,4$ & $86,26 \pm 24,8$ & $84,84 \pm 26,7$ \\
\hline Saúde Mental & $66,78 \pm 7,6$ & $58,56 \pm 20,6$ & $73,41 \pm 16,3$ & $68,36 \pm 17,9$ \\
\hline Média total dos setores & $73,49 \pm 8,75$ & $69,03 \pm 9,9$ & $76,61 \pm 9,1$ & $74,83 \pm 8,3$ \\
\hline Font Auta propra
\end{tabular}

Fonte: Autoria própria (2013).

\section{Discussão}

No presente estudo, o item Vitalidade do questionário SF36, refere-se a cansaço e esgotamento, foi o que apresentou uma das piores notas para todos os setores, com pior nota para o setor I, seguido do setor III e setor II. Hadler (1999) garante que não apenas os fatores físicos acentuam a dor crônica, mas, também, os fatores psicológicos como alguma insatisfação com o trabalho exercido, com o supervisor, com fatores estressores como o tédio e o acúmulo de tarefas. Em estudo semelhante com professores do ensino fundamental, foi 
observado que o domínio vitalidade (46,26 pontos) apresentou o menor escore, o que pode repercutir no estado de saúde desses trabalhadores (ROCHA; FERNANDES, 2008).

As notas encontradas para o quesito Saúde Mental no questionário SF36 não foram altas, sendo no geral de 66,78 pontos. O setor I apresentou as piores notas em todos os itens (58,56 pontos).

O estresse relacionado ao trabalho ou estresse ocupacional pode acarretar diversos prejuízos à saúde dos trabalhadores, incluindo a saúde mental, aumento da pressão arterial e alterações de comportamento, o que pode gerar futuramente em hipertensão, depressão, lesões, entre outros. Este processo de adoecimento surge a partir de algumas fontes, sendo os estressores intrínsecos do trabalho (carga de trabalho, condições físicas de trabalho pobres, turnos, perigo físico); o estresse envolvendo o conflito de papeis e responsabilidade; relação com o grupo de trabalho, entre colegas, chefias e subordinados; plano de carreira e insegurança no trabalho; estrutura organizacional, clima, restrições sobre comportamento, processo de tomada de decisões e por fim as relações entre trabalho, família e vida social (CHEN; WONG; YU, 2009).

A nota baixa encontrada no quesito saúde mental é, no mínimo, preocupante, já que representa a sensação de nervosismo e depressão, de acordo com a descrição do SF36. As notas baixas sempre do mesmo setor I solicita uma investigação juntamente com estratégias de melhoria no sistema de trabalho, abordando as causas desse resultado.

Em estudo realizado com funcionários da limpeza de um hospital público foi observado que o domínio dor apresentou baixo escore (66,9 pontos) para o questionário SF36 (MARTARELLO; BENATTI, 2009). Da mesma forma, no presente estudo, foi encontrado baixo escore para o domínio dor, especialmente no setor de limpeza e manutenção. Esse fato possibilita estabelecer relação entre a atividade exercida e o desconforto identificado, pois este setor realiza seu trabalho na maioria das vezes na posição em pé ou agachada, causando maior fadiga, principalmente em membros inferiores. Em recente estudo publicado por Fontes et al. (2013) foi observado que funcionários que praticam suas atividades na posição ortostática apresentam maiores riscos biomecânicos e posturais quando comparados com os funcionários que trabalham na posição sentada.

O sistema de trabalho pode ser nocivo quando não há uma investigação ampla sobre o que acontece com seus funcionários, causando desequilíbrio, prejuízos e desajuste no trabalho (SMITH; CONWAY; KARSH, 1999). Estes autores defendem que raramente um problema no trabalho acontece devido a um único fator, mas, de uma combinação de diversos elementos. Portanto, para se obter melhora no ambiente de trabalho e na redução de desconforto é necessária uma estratégia de grande abrangência, incluindo diversos aspectos para produção de bem-estar e equilíbrio no trabalho. Côté et al. (2008) afirmam que o ambiente de trabalho pode causar danos psicossociais, tensão no trabalho, insegurança e mau relacionamento com os colegas de trabalho. Além disso, fatores individuais, como idade, educação e gênero também podem influenciar nessas condições.

Os funcionários do presente estudo não passaram por treinamento postural e também não tem nenhum programa de incentivo de melhoria de qualidade de vida. Portanto, as ações baseadas na Promoção de Saúde podem ser forte aliadas no processo de disseminação de novos hábitos saudáveis.

Nos Estados Unidos foi criado o projeto Health People Initiatives que incentivou as empresas a investirem em programas baseados no bem-estar e melhora de estilo de vida de seus funcionários, e ainda a controlarem os gastos com saúde. As empresas que implantaram este programa tiveram como resultado menos riscos de saúde, uma redução em custos com saúde e ainda aumento de produtividade (WILLIAMS; DAY, 2011).

Embora os escores encontrados no SF36 não apresentaram diferença estatística, o estudo aponta diferentes pontuações obtidas em relação aos setores de trabalho. Talvez a 
diferença de remuneração, educação e exposição biomecânica, entre os setores, possam explicar as menores notas apresentadas pelo setor I. Portanto, o baixo poder econômico do setor I, associado às condições de alimentação, transporte, vida social e educação, parecem afetar a qualidade de vida desses funcionários.

A partir desses resultados é possível constatar a necessidade de avaliações periódicas, como a análise ergonômica e de qualidade de vida, para que se possa conhecer a atividade laboral como um todo, desde os seus benefícios até os prejuízos. Com tais procedimentos, o planejamento de ações para transformação do ambiente será mais eficiente e preciso, agindo diretamente no foco do problema.

\section{Referências}

ARTAZCOZ, L. La salud laboral ante los retos de La nueva economía. Gaceta Sanitaria, Barcelona, v. 16, n. 6, nov./dic. 2002.

BRASIL. Ministério da Saúde. As castas da promoção de saúde. Série B: Textos Básicos em Promoção de Saúde. Brasilia - DF. 2002. Disponível em: <http://bvsms.saude.gov.br/ bvs/ publicacoes/cartas_promocao.pdf>. Acesso em: 20 jul. 2011.

CHEN, W. Q.; WONG, T. W.; YU, T. S. Influence of occupational stress on mental health among Chinese off-shore oil workers. Scandinavian Journal of Public Health, v. 37, n. 7, p. 766-773, 2009.

CICONELLI, R. M. et al. Tradução para a língua portuguesa e validação do questionário genérico de avaliação de qualidade de vida SF-36 (Brasil SF-36). Revista Brasileira de Reumatologia, v. 39, n. 3, p. 143-501, mai./jun. 1999.

CÔTÉ, P. et al. The burden and determinants of neck pain in workers: results of the Bone and Joint Decade 2000-2010 Task Force on Neck Pain and Its Associated Disorders. Spine, v. 15, n. 4, p. 60-74, 2008.

FONTES, C. F. et al. Comparação da sobrecarga biomecânica em funcionários que executam suas tarefas na posição ortostática e sentada. Revista Brasileira de Ciência e Movimento, Taguatinga, v. 21, n.1, p. 10-15, 2013.

FREITAS, A. L. P.; SOUZA, R. G. B.; QINTELLA, H. L. M. M. Qualidade de Vida no Trabalho do técnico-administrativo em IES públicas: uma análise exploratória. Revista Brasileira de Qualidade de Vida, Ponta Grossa, v. 5, n. 2, p. 01-12, abr./jun. 2013.

HADLER, N. M. Occupational musculoskeletal disorders. Philadelphia (PA): Lippincott Williams \& Wilkins, 1999.

JARDIM, R.; BARRETO, S. M.; ASSUNÇÃO, A. Á. Condições de trabalho, qualidade de vida e disfonia entre docentes. Cadernos de Saúde Pública, Rio de Janeiro, v. 23, n. 10, p. 2439-2461, out. 2007.

LUGO, L. H. A.; GARCIA, H. I. G; GÓMEZ, C. R. Reliability of SF36 quality of life in health questionnaire in Medellín. Colombia. Revista Nacional Salud Pública, v. 24, n. 2, p. 37-50, jul. 2006. 
MACIEL, E. S. et al. Correlação entre nível de renda e os domínios da qualidade de vida de população universitária brasileira. Revista Brasileira de Qualidade de Vida, Ponta Grossa, v. 5, n. 1, p. 53-62, jan/mar. 2013.

MARTARELLO, N. A.; BENATTI, M. C. C. Qualidade de vida e sintomas osteomusculares em trabalhadores de higiene e limpeza hospitalar. Revista da Escola de Enfermagem da USP, São Paulo, v. 43, n. 2, p. 422-428, jun. 2009.

MARTINEZ, M. C. As relações entre satisfação com aspectos psicossociais no trabalho e a saúde do trabalhador. 2002. 243 f. Dissertação (Mestrado em Saúde Ambiental) Programa de Pós-Graduação em Saúde Ambiental, Faculdade de Saúde Pública, Universidade de São Paulo, São Paulo.

OLIVEIRA, S. A. qualidade da qualidade: uma perspectiva em Saúde do Trabalhador. Cadernos de Saúde Pública, Rio de Janeiro, v. 13, n. 4, p. 625-634, out./dez. 1997.

RIBEIRO, C. V. S.; LEDA, D. B. O significado do trabalho em tempos de reestruturação produtiva. Estudos e Pesquisas em Psicologia, Rio de Janeiro, v. 4, n. 2, p. 76-83, 2004.

ROCHA, V. M.; FERNANDES, M. H. Qualidade de vida de professores do ensino fundamental: uma perspectiva para a promoção da saúde do trabalhador. Jornal Brasileiro de Psiquiatria, Rio de Janeiro, v. 57, n. 1, p. 23-27, 2008.

SMITH, M. J; CONWAY, F. T; KARSH, B. T. Occupational stress in human computer interaction. Industrial Health, v. 37, n. 2, p. 157-173, 1999.

WILLIAMS, L.; DAY, B. T. Medical cost savings for web-based wellness program participants from employers engaged in health promotion activities. American Journal of Health Promotion, Pennsylvania, v. 25, n. 4, p. 272-280, 2011. 